\title{
Effets des composts à base de déchets d'agrumes et du tourteau de karité sur la disponibilité du phosphore du sol : une étude en conditions contrôlées
}

\author{
Sanonka TCHEGUENI ${ }^{1 *}$, Koffi A. KILI ${ }^{1}$, Magnoudéwa BODJONA ${ }^{1}$, \\ Moursalou KORIKO ${ }^{1}$, Mohamed HAFIDI ${ }^{2}$, Gnon BABA ${ }^{1}$ et Gado TCHANGBEDJI ${ }^{1}$ \\ ${ }^{1}$ Laboratoire GTVD (Gestion, Traitement et Valorisation des Déchets), Faculté des Sciences, \\ Université de Lomé ; BP 1515 Lomé, Togo. \\ ${ }^{2}$ Laboratoire d'Ecologie et Environnement, Faculté des Sciences Semlalia, Université Cadi Ayyad; \\ B.P.2390 Marrakech, Maroc. \\ *Auteur correspondant, E-mail: tchegsani@live.fr, Tél : 00228-90939987
}

\section{RESUME}

Le compost, riche en matière organique, contient des éléments fertilisants tels que le phosphore, l'azote et le potassium. La biodisponibilité de ces éléments fertilisants du compost dans le sol dépend de la minéralisation de la matière organique mais aussi des réactions de fixation de ces éléments. Ce travail vise à étudier la disponibilité du phosphore de deux composts : le compost CA obtenu à base des déchets d'agrumes et le compost CK obtenu à base du tourteau de karité. Les composts sont mélangés au sol à raison de $0,5 \mathrm{~g}$ de compost pour $50 \mathrm{~g}$ de sol et sont incubés dans des conditions contrôlées pendant 180 jours. L'humidité a été ajustée à $2 / 3$ de la capacité au champ et la température à $28^{\circ} \mathrm{C}$. Entre le début et 180 jours d'incubation, une augmentation de 33, 25 et $-2 \%$ de la teneur en phosphore assimilable est notée pour les traitements sol+CA, sol+CK et le témoin respectivement et près de $85 \%$ du phosphore des composts sont disponibles. L'étude de la dynamique du phosphore montre qu'une partie du phosphore s'immobilise sous forme de phosphore lié à l'aluminium. Ceci est plus notable dans le traitement avec le compost CK.

(C) 2012 International Formulae Group. All rights reserved.

Mots clés : disponibilité du phosphore, compost, sol, conditions contrôlées.

\section{INTRODUCTION}

Le compostage est l'un des moyens de valorisation des déchets. Outre l'amélioration des propriétés physiques, chimiques et biologiques (Aggelides et al., 2000; N'Dayegamiye et al., 2005), le compost apporte aussi au sol les éléments minéraux tels que le phosphore (McDowell et Sharpley, 2004), l'azote et les oligo-éléments. Le phosphore est l'un des éléments essentiels dans la nutrition des plantes. Il représente approximativement 0,2 à $0,5 \%$ de la masse sèche des composts d'origine urbaine
(Koledzi et al., 2011). Cette teneur peut avoisiner $1,9 \%$ dans les composts avec déjection animale (ADEME, 2001). Les travaux de Takeda (2009) montrent que l'ajout de compost au sol augmente la quantité de phosphore disponible pour les plantes. La disponibilité du phosphore du compost dans le sol dépend de la minéralisation de la matière organique mais aussi des réactions de sa fixation. En milieux très acides ou très basiques, les phosphates évoluent vers des formes cristallisées insolubles et par conséquent, soustraites au cycle biologique. 
Ce phénomène dit de rétrogradation peut-être atténué par la matière organique du compost constituée en grande partie des substances humiques (Zaharah et Bah, 1997 ; Atiyeh et al., 2002), qui complexent les cations métalliques et libèrent ainsi les ions hydrogéno-phosphates, la forme assimilée par les plantes. La stimulation de l'activité microbienne par la présence de la matière organique augmente aussi la biodisponibilité du phosphore du sol (Takeda, 2009).

Les composts de déchets agroalimentaires sont exempts de germes pathogènes, de métaux lourds, donc sont de meilleure qualité par rapport aux composts de déchets urbains (ADEME, 2001; Koledzi et al., 2011). Les composts faisant l'objet de cette étude proviennent du compostage des déchets d'agrumes provenant de la fabrication de jus de citron, d'orange et de pamplemousse et du tourteau de karité. Le tourteau de karité est un déchet d'une huilerie, la nouvelle industrie des oléagineux du Togo.

L'objectif de cette étude est de déterminer la valeur fertilisante phosphatée de ces composts à court terme dans le sol afin de prévoir la quantité de composts à apporter au sol pour assurer une nutrition optimale des plantes. L'effet des composts sur la dynamique du phosphore dans le sol fait également partie de notre préoccupation.

\section{MATERIEL ET METHODES Composts}

Deux composts élaborés à base de déchets agro-alimentaires avec des déjections de caprins, pendant six mois ont fait l'objet de cette étude :

CA : compost élaboré à partir des déchets d'agrumes (déchets issus de la production de jus d'agrumes) et du fumier (Tchegueni et al., 2010).

- $\quad$ CK : compost élaboré à partir du tourteau de karité et du fumier (Tchegueni, 2011).

Les caractéristiques physico-chimiques de ces composts sont dans le Tableau 1.
Sol

Le sol utilisé dans cette étude est un sol d'apport colluvionnaire dont les caractéristiques sont consignées dans le Tableau 2.

\section{Incubation en conditions contrôlées}

Un mélange de $50 \mathrm{~g}$ de sol et $0,5 \mathrm{~g}$ de compost est placé dans un flacon de $75 \mathrm{ml}$, et l'humidité a été corrigée afin d'atteindre les 2/3 de la capacité au champ. Ensuite, les flacons ont été placés dans des enceintes étanches de $500 \mathrm{ml}$. A l'intérieur de chaque enceinte, un flacon de $20 \mathrm{ml}$ contenant $10 \mathrm{ml}$ d'eau déminéralisée est placé, afin d'assurer la saturation en eau de l'atmosphère pour éviter que les échantillons deviennent secs (Gagnon, 2004; Javier, 2005; Som, 2006). L'ensemble est maintenu à $28^{\circ} \mathrm{C}$. Les flacons sont ouverts toutes les semaines pour l'aération du milieu. Le plan expérimental est formé de trois traitements (témoin (sol seul), sol+CA, sol+CK) et de trois répétitions de chaque traitement.

Cette dose correspond à environ 33 tonnes de compost par hectare en considérant la densité apparente du sol qui est 1,65 et un horizon 0-20 cm.

\section{Analyses physico-chimiques pH}

La mesure du pH est effectuée sur une suspension de $10 \mathrm{~g}$ de sol dans $25 \mathrm{ml}$ d'eau distillée. Après dix minutes d'agitation et une heure de repos, le pH est mesuré à l'aide d'un pH-mètre à électrode Crison pH 25.

\section{Phosphore}

Le phosphore assimilable est déterminé par la méthode d'Olsen (Mathieu, 2003). Cette méthode détermine le phosphore alcalino-soluble extrait par $\mathrm{NaHCO}_{3}$ à $\mathrm{pH}=$ 8,5 .

Le fractionnement $\mathrm{du}$ phosphore minéral est effectué par la méthode de Chang et Jackson (1957). Cette méthode a été légèrement modifiée pour exclure les phosphates de fer et d'aluminium d'inclusion. Sur un échantillon de sol, le phosphore soluble, le phosphore lié à l'aluminium, le 
phosphore lié au fer et le phosphore lié au calcium sont successivement extraits par la solution $\mathrm{NH}_{4} \mathrm{Cl} 1 \mathrm{M}$, la solution de $\mathrm{NH}_{4} \mathrm{~F} 0,5$ $\mathrm{M}(\mathrm{pH}=7)$, la solution de $\mathrm{NaOH} 0,1 \mathrm{M}$ et la solution de $\mathrm{H}_{2} \mathrm{SO}_{4} 0,25 \mathrm{M}$.

Dans tous les cas, le phosphore est dosé par la méthode du bleu de phospho-molybdate à $660 \mathrm{~nm}$, en utilisant le Spectrophotomètre Genesys UV-Vis10.

\section{Traitement statistique des données}

La valeur moyenne de chaque traitement, ainsi que la variance et l'écart-type correspondant, ont été calculés en tenant compte des trois répétitions réalisées. Les données ainsi obtenues ont fait l'objet d'analyse statistique avec le logiciel Assistat Version 7.5 Bêta (2010). L'analyse des variances (ANOVA) a été réalisée. Dans les cas où des différences significatives ont été trouvées $(\mathrm{p}<0,05)$, les moyennes ont été séparées en utilisant le test de Turkey ( $\mathrm{p}<$ $0,05)$.

\section{RESULTATS}

La teneur en phosphore $\left(\mathrm{P}_{2} \mathrm{O}_{5}\right)$ est de $1,17 \%$ pour le compost $\mathrm{CK}$ et de $1,98 \%$ pour CA. Ces composts ont leur teneur en phosphore supérieure à celle des composts urbains rencontrés au Togo et au Mali. Au Togo, Koledzi (2011) a trouvé pour les composts des déchets urbains de Lomé, une teneur en $\mathrm{P}_{2} \mathrm{O}_{5}$ de $0,8-1,36 \%$ de matière sèche. Au Mali, Soumaré et al. (2003) ont trouvé $0,09 \%$.

Le $\mathrm{pH}$ du compost à base de déchets d'agrumes CA est de 7,24; tandis que le compost à base de tourteau de karité $\mathrm{CK}$ est légèrement acide $(\mathrm{pH}=6,52)$. Le sol ayant servi à cette étude est un sol sablonneux de $\mathrm{pH}=7,73$. C'est un sol pauvre en matière organique dont la teneur en carbone est environ $0,6 \%$.

\section{Effets des composts sur le $\mathbf{p H}$ du sol}

Les valeurs du $\mathrm{pH}$ mesurées au cours de l'incubation sont consignées dans le Tableau 3. L'apport du compost des déchets d'agrumes $\mathrm{CA}$ au sol a relevé son $\mathrm{pH}$ de 0,15 unité alors que dans le cas du compost du tourteau de karité $\mathrm{CK}$, son $\mathrm{pH}$ a diminué de 0,5 unité. L'observation de ces données montre que le $\mathrm{pH}$ du traitement sol+CK et du témoin (sol) n'ont pas significativement varié tandis que celui du traitement sol+CA a baissé, entre le début et la fin de l'expérience.

\section{Effet sur le phosphore assimilable}

Les teneurs en phosphore assimilable, déterminées au cours de cette étude par la méthode d'Olsen, sont consignées dans le Tableau 4. Au niveau du témoin (sol sans compost), cette teneur ne varie pas significativement au cours de l'incubation. L'ajout des composts, à la dose de l'expérience $(50 \mathrm{~g}$ de sol pour $0,5 \mathrm{~g}$ de compost), a amélioré sensiblement la teneur en phosphore assimilable dans le sol (près de $393 \%$ pour le traitement sol+CA et $243 \%$ pour sol+CK). Une augmentation significative de la teneur en phosphore assimilable entre le début et la fin de l'expérience a été notée: une augmentation de $33 \%$ pour le traitement sol+CA et $25 \%$ pour sol+CK contre une réduction de $2 \%$ pour le témoin. On note donc un phénomène de rétrogradation du phosphore dans le témoin.

Pour mieux comprendre l'évolution du phosphore dans le sol, nous avons procédé au fractionnement des formes minérales du phosphore des traitements en utilisant la méthode de Chang et Jackson (1957), modifiée pour ne pas tenir compte des formes protégées par les oxydes de fer et d'aluminium (phosphore d'inclusion).

\section{Fractionnement du phosphore}

Le phosphore soluble et le phosphore peu soluble (phosphore lié à l'aluminium, phosphore lié au fer et phosphore lié au calcium) sont déterminés au cours de l'étude. Le phosphore lié au fer est très faible dans tous les traitements.

La fraction soluble est extraite par la solution de chlorure d'ammonium. Elle est analogue à la quantité de phosphore disponible extraite par la méthode d'Olsen à quelques exceptions près: elle est plus 
importante dans le traitement sol+CA et moins dans le traitement sol+CK.

Les formes insolubles du phosphore sont des formes cristallisées : le phosphore lié $\mathrm{au}$ fer $(\mathrm{P}-\mathrm{Fe})$, à l'aluminium $(\mathrm{P}-\mathrm{Al})$ et au calcium (P-Ca). Le P-Fe et le $\mathrm{P}-\mathrm{Al}$ sont plus importants dans le traitement sol+CK que dans le traitement sol+CA (Figures 2 et 3).
Quant au phosphore lié au calcium, il est plus important dans le traitement sol+CA. La teneur en (P-Ca) dans le sol a augmenté de près de $1 \%$ après six mois d'incubation. Une réduction de $2,3 \%$ et $3,5 \%$ de la teneur en (P$\mathrm{Ca}$ est notée dans le traitement sol+CA et dans le traitement sol+CK respectivement (Figure 4).

Tableau 1: Composition chimique des deux composts (moyenne par rapport à la masse sèche士écart-type).

\begin{tabular}{lcc}
\hline & CA & CK \\
\hline $\mathrm{pH}$ & $7,24 \pm 0,05$ & $6,52 \pm 0,10$ \\
$\mathrm{MO}(\%)$ & $59,50 \pm 0,40$ & $75,50 \pm 1,64$ \\
$\mathrm{~N}(\%)$ & $3,78 \pm 0,26$ & $4,20 \pm 0,15$ \\
$\mathrm{C} / \mathrm{N}$ & 8,37 & 9,18 \\
$\mathrm{P}_{2} \mathrm{O}_{5}(\%)$ & $1,98 \pm 0,14$ & $1,17 \pm 0,07$ \\
$\mathrm{~K}_{2} \mathrm{O}(\%)$ & $2,87 \pm 0,44$ & $2,02 \pm 0,17$ \\
$\mathrm{CaO}(\%)$ & $2,82 \pm 0,18$ & $1,99 \pm 0,31$ \\
$\mathrm{MgO}(\%)$ & $1,04 \pm 0,11$ & $0,50 \pm 0,09$ \\
\hline \multicolumn{2}{c}{ MO : matière organique }
\end{tabular}

Tableau 2: Caractéristiques physico-chimiques du sol (moyenne par rapport à la masse sèche \pm écart-type).

\begin{tabular}{lclc}
\hline $\mathbf{p H}_{\text {eau }}$ & $\mathbf{7 , 7 3 \pm 0 , 2 4}$ & \multicolumn{2}{c}{ Granulométrie (\%) } \\
\hline $\mathrm{COT}(\%)$ & $0,595 \pm 0,066$ & Sable grossier & 60,45 \\
$\mathrm{P}_{\text {olsen }}(\mathrm{mg} / \mathrm{kg})$ & $13,016 \pm 0,572$ & Sable fin & 25,15 \\
$\mathrm{~K}_{\text {éch. }}(\mathrm{mg} / \mathrm{kg})$ & $35,200 \pm 2,406$ & Limon grossier & 2,75 \\
$\mathrm{Ca}_{\text {éch. }}(\mathrm{mg} / \mathrm{kg})$ & $336,245 \pm 16,172$ & Limon fin & 3 \\
$\mathrm{Mg}_{\text {éch. }}(\mathrm{mg} / \mathrm{kg})$ & $27,200 \pm 5,503$ & Argile & 7 \\
$\mathrm{CEC}\left(\mathrm{cmol}^{+} / \mathrm{kg}\right)$ & $1,80 \pm 0,09$ & & \\
\hline \multicolumn{2}{c}{$\mathrm{COT}:$ carbone organique total } \\
\multicolumn{2}{c}{ CEC : capacité d'échange cationique } & &
\end{tabular}

Tableau 3: pH des différents traitements en fonction de la durée de l'incubation.

\begin{tabular}{cccc}
\hline Durée & Sol & Sol + CA & Sol + CK \\
\hline $0 \mathrm{j}$ & $7,73 \mathrm{a}$ & $7,88 \mathrm{a}$ & $7,33 \mathrm{a}$ \\
$30 \mathrm{j}$ & $7,88 \mathrm{a}$ & $7,72 \mathrm{ab}$ & $7,61 \mathrm{a}$ \\
$90 \mathrm{j}$ & $7,73 \mathrm{a}$ & $7,39 \mathrm{~b}$ & $7,32 \mathrm{a}$ \\
$180 \mathrm{j}$ & $7,71 \mathrm{a}$ & $7,48 \mathrm{~b}$ & $7,32 \mathrm{a}$ \\
$S M D$ & 0,37 & 0,37 & 0,38 \\
\hline
\end{tabular}

$S M D=$ plus petite différence significative. Les moyennes suivies de la même lettre dans la même colonne ne diffèrent pas significativement entre elles selon le test de Turkey à $p<0,05$. 
Tableau 4: Teneur en phosphore assimilable (selon la méthode d'Olsen) des différents traitements en fonction de la durée d'incubation ( $\mathrm{mg} / \mathrm{kg} \mathrm{MS})$.

\begin{tabular}{lccc}
\hline Durée & Sol & Sol+CA & Sol+CK \\
\hline $0 \mathrm{j}$ & $13,016 \mathrm{a}$ & $64,116 \mathrm{c}$ & $44,627 \mathrm{c}$ \\
$30 \mathrm{j}$ & $15,079 \mathrm{a}$ & $70,309 \mathrm{c}$ & $47,905 \mathrm{bc}$ \\
$90 \mathrm{j}$ & $13,492 \mathrm{a}$ & $77,778 \mathrm{~b}$ & $55,373 \mathrm{ab}$ \\
$180 \mathrm{j}$ & $12,698 \mathrm{a}$ & $85,246 \mathrm{a}$ & $55,920 \mathrm{a}$ \\
$S M D$ & 4,098 & 7,110 & 7,773
\end{tabular}

$S M D=$ plus petite différence significative. Les moyennes suivies de la même lettre dans la même colonne ne diffèrent pas significativement entre elles selon le test de Turkey à $p<0,05$.

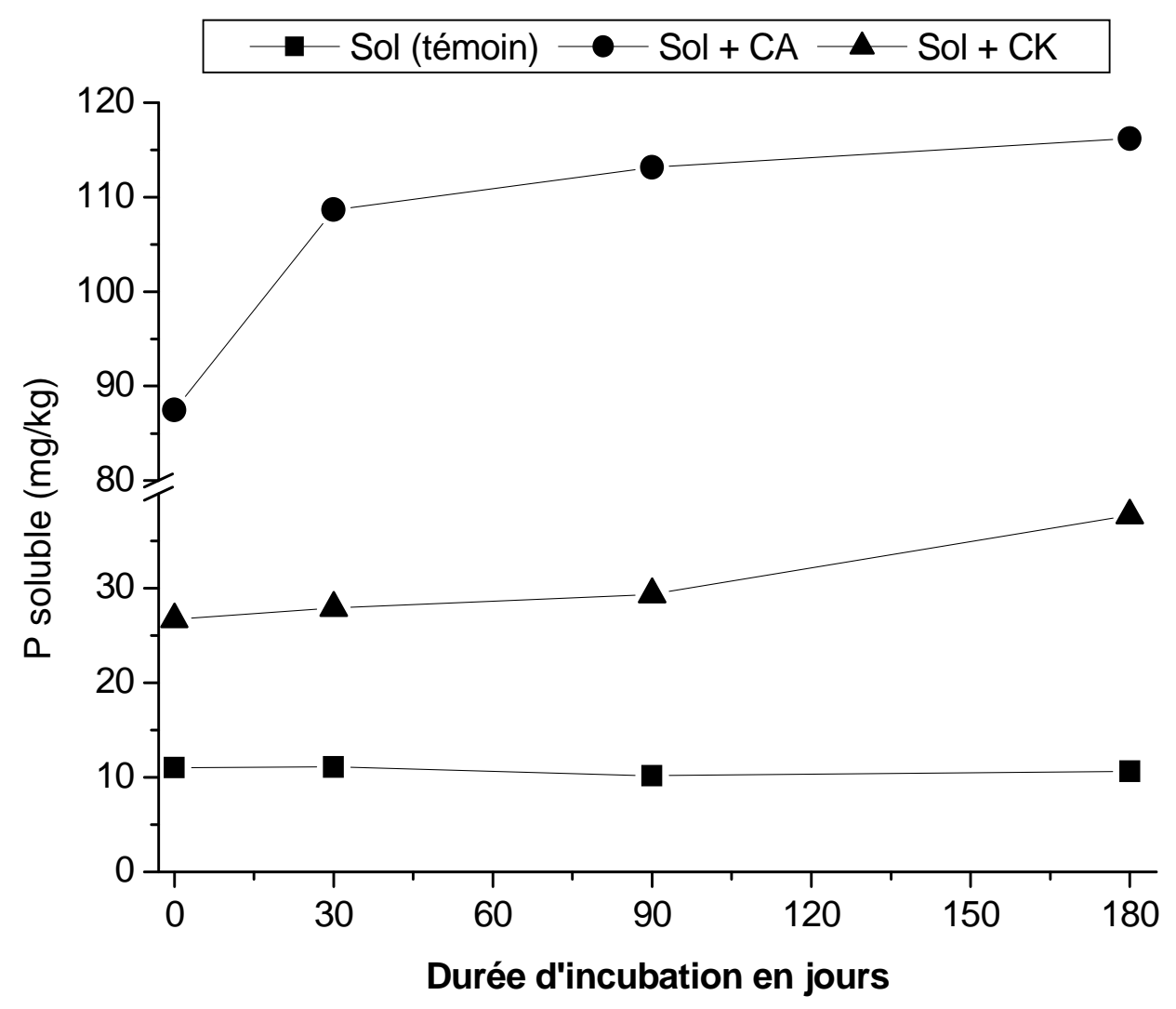

Figure 1 : Evolution du phosphore soluble des différents traitements. 


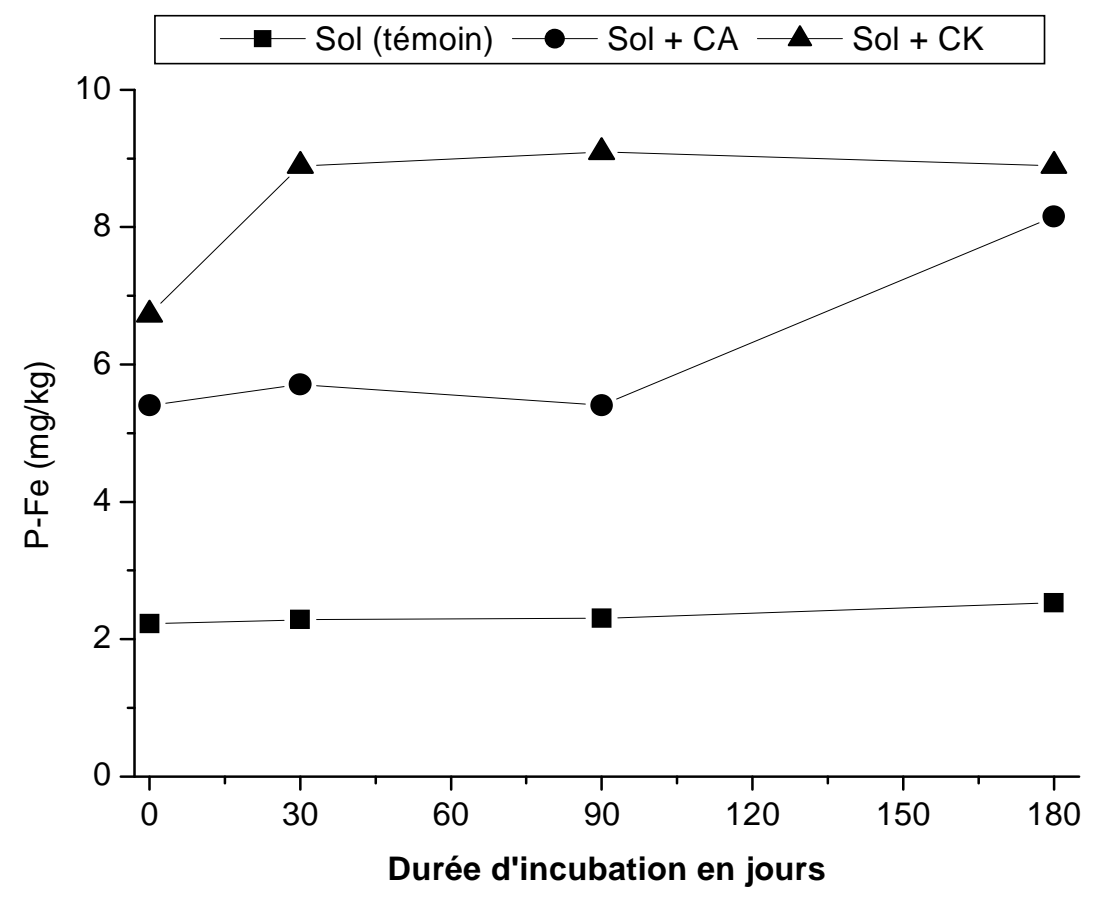

Figure 2 : Evolution du phosphore lié au fer des différents traitements.

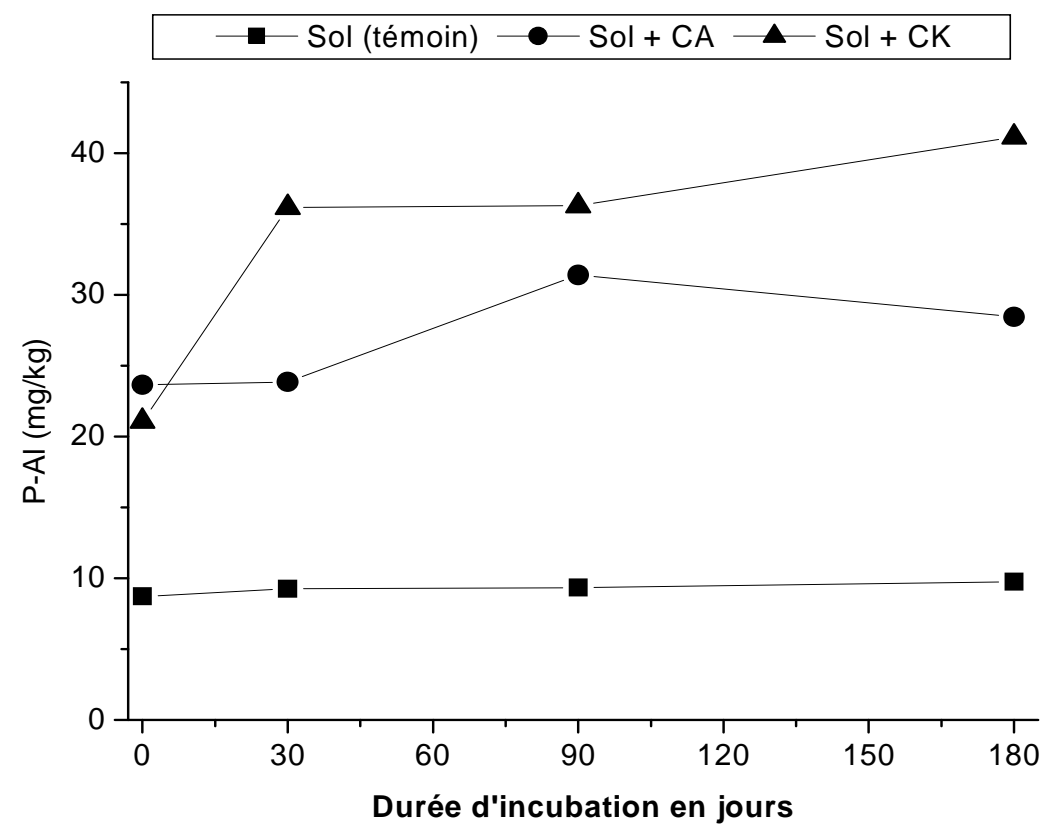

Figure 3 : Evolution du phosphore lié à l'aluminium des différents traitements. 


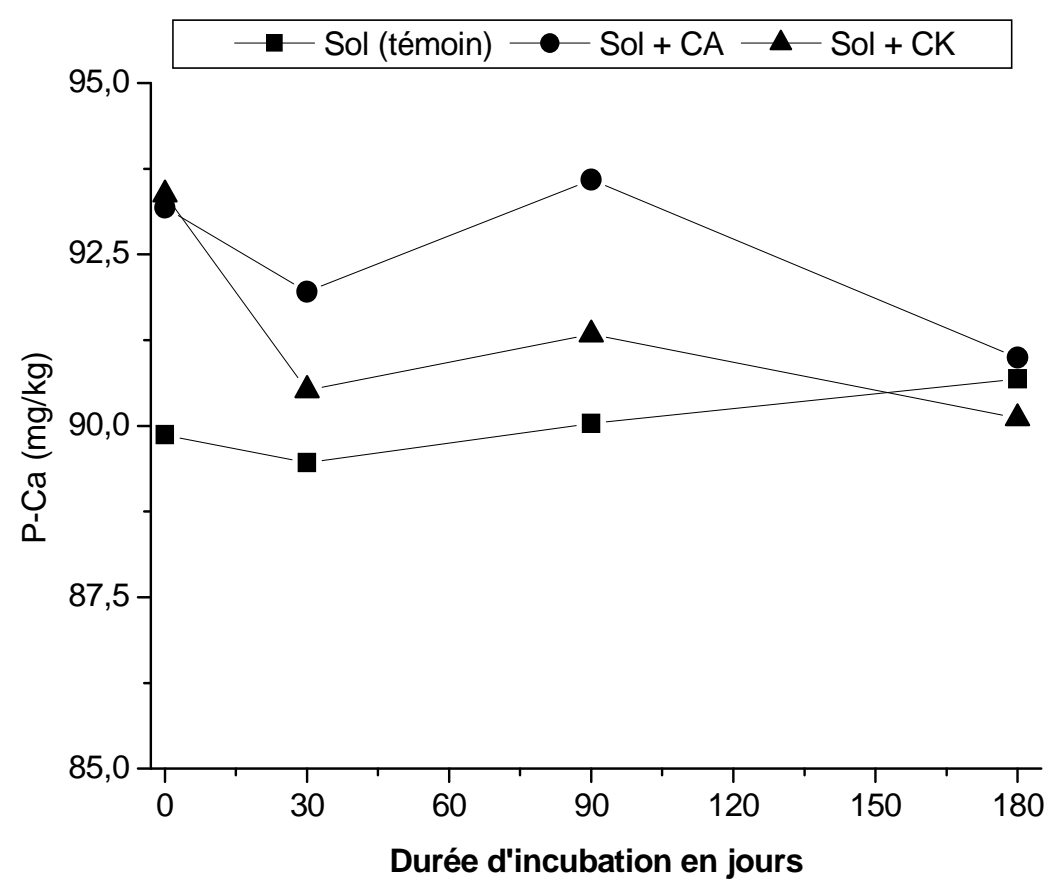

Figure 4 : Evolution du phosphore lié au calcium des différents traitements.

\section{DISCUSSION}

Le compost apporté au sol affecte la variation $\mathrm{du} \mathrm{pH}$ de ce dernier. Ainsi le caractère acide du compost $\mathrm{CK}$ a contribué à abaisser le $\mathrm{pH}$ du sol de 0,5 unité. La diminution du $\mathrm{pH}$ à la fin de l'incubation du traitement sol+CA serait due à la minéralisation de la matière organique avec accumulation du $\quad \mathrm{CO}_{2}$ dans le bocal expérimental.

Les composts ont apporté du phosphore total au sol car ils en contiennent à plus de $1 \%$ de leur masse sèche. Cet apport a amélioré la teneur en phosphore assimilable du sol. La minéralisation de la forme organique du phosphore et les effets de la matière organique sur le phosphore du sol ont contribué à l'augmentation de la forme disponible du phosphore au cours de l'incubation. En effet, la stimulation de l'activité microbienne par la présence de la matière organique augmente la bio-disponibilité du phosphore du sol (Takeda, 2009). La complexation des cations métalliques du sol par les substances humiques du compost réduit la fixation du phosphore sur les cations $\mathrm{Al}^{3+}, \mathrm{Fe}^{2+}$ et $\mathrm{Ca}^{2+}$, augmentant donc sa disponibilité aux plantes (Zaharah et Bah, 1997 ; Atiyeh et al., 2002). Dans le sol sans compost, le phénomène de rétrogradation du phosphore peut être dû au manque de matière organique. L'évolution du phosphore lié au calcium (Figure 4) explique ce phénomène : le phosphore disponible se lie aux $\mathrm{Ca}^{2+}$ pour former le phosphate tricalcique. $\mathrm{La}$ teneur en P-Ca (Figure 4) augmente dans le sol sans compost et diminue dans les traitements sol+compost. Cette baisse au niveau des traitements sol+compost peut-être attribuée à la complexation des ions $\mathrm{Ca}^{2+}$ par les substances humiques contenues dans le compost ; ce qui contribue à la solubilisation des phosphates apatitiques du sol par formation de chélates des ions métalliques des apatites (Thien et Myers, 1992). La fraction du phosphore lié au fer ou celle liée à l'aluminium est plus notable dans le 
traitement sol+CK. Le caractère acide du compost $\mathrm{CK}$ peut contribuer à l'immobilisation du phosphore sous ces formes car dans les milieux acides et riches en oxyhydroxydes, le phosphore se fixe sur les oxydes d'aluminium et l'oxyhydroxyde de fer (Mathieu, 2003) et se précipite.

Le phosphore des composts est très disponible dans le sol. Déterminée en tenant compte de la contribution des composts en phosphore total et en phosphore assimilable du sol, au bout de six mois, cette disponibilité est de $84 \%$ pour le compost CA et $85 \%$ pour le compost CK. Ceci sans compter le phosphore immobilisé par les microorganismes du sol, qui sera disponible après la mort des microorganismes et la minéralisation de leur matière organique. Cette disponibilité est meilleure comparée à celle trouvé par Mustin (1987) au cours de ses travaux. En effet, il a trouvé que 50 à $60 \%$ du phosphore des composts sont disponibles au cours de la première année d'épandage. La disponibilité du phosphore des composts dans le sol dépend donc de la composition chimique des composts, des caractéristiques physico-chimiques du sol. Aussi, plusieurs méthodes peuvent être utilisées pour déterminer le phosphore assimilable. Nous avons utilisé la méthode d'Olsen dans notre cas tandis que les méthodes comme la méthode de Dyer, de Bray et de Méhlich peuvent être utilisées.

\section{Conclusion}

Les composts à base de déchets d'agrumes (CA) et du tourteau de karité (CK) ont un effet notable sur la disponibilité du phosphore du sol. Le compost CA a un potentiel fertilisant phosphaté plus élevé que le compost CK. Le compost CA a amélioré, à la dose expérimentale, la teneur en phosphore assimilable de $393 \%$ tandis que le compost CK de 243\%. L'augmentation de la teneur en phosphore disponible au bout de six mois dans les conditions contrôlées est de $33 \%$ pour le traitement sol+CA, $25 \%$ pour sol+CK et $-2 \%$ pour le témoin. Le coefficient de disponibilité $\mathrm{du}$ phosphore au bout de six mois des composts est voisin de $85 \%$. La disponibilité du phosphore des composts dans le sol dépend de la nature du sol, du type de culture et des conditions climatiques. Ce travail, réalisé au laboratoire dans les conditions contrôlées, ne permet de formuler que des hypothèses sur la possibilité d'une meilleure prévision du comportement des différents composts dans le sol. Des essais au champ avec de divers types cultures permettront d'évaluer l'efficacité des différents composts à court et long terme.

\section{REFERENCES}

ADEME. 2001. Approche de la qualité des composts de déchets en France. Résultat d'une enquête en 1998.

Aggelides SM, Londra PA. 2000. Effects of compost produced from town wastes end sewage sludge on the physical properties of a loamy and a clay soil. Bioresource Technology, 71: 253-259.

Atiyeh RM, Lee S, Edwards CA, Arancon NQ, Metzger JD. 2002. The influence of humic acids derived from earthwormprocessed organic wastes on plant growth. Bioresource Technology, 84: 714.

Chang SC, Jackson ML. 1957. Fractionation of soil phosphorus. Soil Science, 84: 133144.

Gagnon B. 2004. Détermination en condition contrôlée de la disponibilité des éléments nutritifs des composts produits à la ferme. Agrosol, 15(1): 10-17.

Javier DS. 2005. Spéciation du Carbone, de 1'Azote et du Phosphore de différentes boues de stations d'épuration au cours de leurs incubations contrôlées dans deux types de sol. Thèse de Doctorat, INPToulouse, France.

Koledzi KE, Baba G, Tchangbedji G, Agbeko K, Matejka G, Feuillade G, Bowen J. 2011. Experimental study of urbans waste composting and evaluation of its agricultural valorization in Lomé (Togo). Asian Journal of Applied Sciences, 4(4): 378-391.

Mathieu C. 2003. Analyse Chimique des Sols, Méthodes Choisies. TEC\&DOC.

McDowell RW, Sharpley AN. 2004. Variation 
of phosphorus leached from Pennsylvanian soils amended with manures, composts or inorganic fertilizer. Agriculture, Ecosystems \& Environment, 102(1): 17-27.

Mustin M. 1987. Le Compost : Gestion de la Matière Organique. Ed. François Dubusc: Paris.

N'Dayegamiye A, Turcot M, Laverdière MR. 2005. Effets d'apports de composts de résidus verts urbains sur les rendements et la nutrition azotée du maïs-grain et sur certaines propriétés du loam argileux de la série Providence. Agrosol, 16(1): 9199.

Som M-P. 2006. Etude moléculaire des composés organiques de compost: formation, transformation dans les sols et action sur les propriétés des sols. Thèse de Doctorat Université de Poitiers, France.

Soumaré M, Tack FMG, Verloo MG. 2003. Effect of municipal solid waste compost and minéral fertilization on plant bowen growth in two tropical agricultural soils of Mali. Bioresource Technology, 86: 1520.
Takeda M, Nakamoto T, Miyazawa K, Murayama T, Okada H. 2009. Phosphorus availability and soil biological activity in an Andosol under compost application and winter cover cropping. Applied Soil Ecology, 42(2): 86-95.

Tchegueni S. 2011. Contribution à la valorisation des déchets agro-alimentaires en compost: Caractérisation physicochimique des composts et étude de leur minéralisation dans deux sols agricoles du Togo. Thèse de doctorat, Université de Lomé, Togo.

Tchegueni S, Kili K, Bodjona M, Tchangbedji G, Baba G, Hafidi M. 2010. Valorisation par compostage des déchets d'agrumes issus des préparations de jus: Caractérisation physico-chimique. Bulletin d'Information de la SOACHIM, 007: 6169.

Thien SJ, Myers R. 1992. Determination of bioavailable phosphorus in soil. Soil Sci. Soc. Am. J., 56: 814-818.

Zaharah AR, Bah AR. 1997. Effect of green manures on $\mathrm{P}$ solubilization and uptake from phosphate rocks. Nutrient Cycling in Agroecosystems, 48: 247-255. 